\title{
THE EULER CHARACTERISTICS OF SU(3)-INSTANTON AND MODULI SPACES OVER FOUR SPHERE
}

\author{
YOULIANG TIAN
}

Moduli spaces of instantons have been proven to possess many remarkable properties. Apart from the celebrated Donaldson theory, there has been successful work done along the line of the Atiyah-Jones Conjecture. Notable results are those obtained by Taubes $[\mathrm{T}]$, and by Boyer, Hurtubise, Mann and Milgram [BHMM]. Some interesting phenomena like Bott periodicity [ $\mathrm{Ti}]$ have also been observed by studying the moduli spaces over the round four sphere. Yet, a more subtle property is perhaps the modularity of partition functions which can be constructed from Euler characteristics of these moduli spaces (or more precisely, $N=4$ topologically twisted supersymmetric Yang-Mills in general) and which was studied and verified in some cases by Vafa and Witten [VW]. Partially motivated by this recent work of Vafa and Witten we will compute the Euler characteristics of $\mathcal{M}_{k}(2)$ and $\mathcal{M}_{k}(3)$ in this short note. Here $\mathcal{M}_{k}(n)$ denotes the moduli space of $S U(n)$-instantons over $S^{4}$ with second Chern class $k$. Since $\mathcal{M}_{k}(2)$ is of odd dimension, calculation of the Euler characteristics of $\mathcal{M}_{k}(3)$ is more desirable for all of these moduli spaces (with $k>1$ ) are of even dimension. Unfortunately, due to the fact that the moduli spaces considered here are not compact (and the compactifications used here are not smooth), our calculations may not be directly used to check the modularity property mentioned above. However, we hope that the present calculation may still be useful. The main results proved in this note are

Theorem [A] (Furuta). $\chi\left(\mathcal{M}_{k}(2)\right)=E(k)$.

Theorem [B]. $\chi\left(\mathcal{M}_{k}(3)\right)=2 E(k+1)+(2 k-3) E(k)-4$ for $k>1$.

In the above theorems, $\chi(M)$ denotes the Euler characteristic of $M$ and $E(k)$ denotes the number of divisors of $k$. The situation for general $\mathcal{M}_{k}(n)$ is more involved, and we hope to return to this and the related problems (e.g. compactifications) in a future paper. Our strategy to prove

Received June 13, 1996.

This work is partially supported by an NSF postdoctoral fellowship. 
the theorems is very simple: we study a circle action on the moduli spaces and derive concrete description of the fixed point set. The proofs are quite elementary.

Remark. From the two theorems above one can check that the partition functions appeared in [VW] fail to be modular which resembles in some sense the holomorphy anomaly discussed in $[\mathrm{VW}]$ when the base manifold is $\mathbb{C P}^{2}$.

To begin the proof we recall a theorem of Donaldson $[\mathrm{D}]$.

Theorem [D]. The moduli space $\mathcal{M}_{k}(n)$ can be defined as the quotient of the set of matrices $\left(\alpha_{1}, \alpha_{2}, a, b\right) \in M_{k \times k}(\mathbb{C}) \times M_{k \times k}(\mathbb{C}) \times M_{n \times k}(\mathbb{C}) \times$ $M_{k \times n}(\mathbb{C})$ satisfying:

$$
\left[\alpha_{1}, \alpha_{2}\right]+b a=0
$$

$$
\text { For all } \lambda, \mu \in \mathbb{C},\left(\begin{array}{c}
\alpha_{1}+\lambda \\
\alpha_{2}+\mu \\
a
\end{array}\right) \text { and }\left(\begin{array}{c}
\alpha_{1}+\lambda \\
-\alpha_{2}-\mu \\
b
\end{array}\right) \text { have maximal rank } k
$$

by the natural action of $(A, g) \in S U(n) \times_{\mathbb{Z}_{n}} G L(k, \mathbb{C})$ :

$$
\alpha_{i} \mapsto g \alpha_{i} g^{-1}, a \mapsto A a g^{-1}, b \mapsto g b A^{-1}
$$

In the theorem above, $\mathbb{Z}_{n}$, the center of $S U(n)$ acts diagonally on $S U(n) \times G L(k, \mathbb{C})$. Although Donaldson [D] only gave the description for based moduli spaces $\mathcal{M}_{k}^{b}$, the results immediately yield the above description for $\mathcal{M}_{k}(n)$. An important fact to keep in mind is that $\mathcal{M}_{k}^{b}(n)$ is smooth and complex algebraic and that $\mathcal{M}_{k}$ is obtained as the quotient of $\mathcal{M}_{k}^{b}$ by the action of $S U(n)$ as in the theorem. The center $\mathbb{Z}_{n}$ of $S U(n)$ acts trivially and $S U(n) / \mathbb{Z}_{n}$ acts freely on the subspace of $\mathcal{M}_{k}^{b}$ where gauge equivalent connections are all irreducible. As a result, $\mathcal{M}_{k}(2)$ are smooth, whereas $\mathcal{M}_{k}(n)$ are all singular for $k \geq n>2$. Now let the circle group $T$ act on $\mathcal{M}_{k}(n)$ by

$$
\left[\left(\alpha_{1}, \alpha_{2}, a, b\right)\right] \mapsto\left[t\left(\alpha_{1}, \alpha_{2}, a, b\right)\right]
$$

where $t=e^{i \theta} \in T,\left[\left(\alpha_{1}, \alpha_{2}, a, b\right)\right] \in \mathcal{M}_{k}$. Here the bracket stands for the equivalence classes under the action of $S U(n) \times_{\mathbb{Z}_{n}} G L(k, \mathbb{C})$. Now we shall study the fixed point set of this action. 


\section{The $\mathrm{SU}(2)$ case}

Since $\mathcal{M}_{1}(2)$ is a five ball, we shall only discuss $\mathcal{M}_{k}(2)$ for $k>1$. Assume $\left[\left(\alpha_{1}, \alpha_{2}, a, b\right)\right]$ is a fixed point of the circle action. Then there exists one parameter subgroup $\left(A_{t}, g_{t}\right) \in S U(2) \times_{\mathbb{Z}_{n}} G L(k, \mathbb{C})$ and a representative $\left(\alpha_{1}, \alpha_{2}, a, b\right)$ of $\left[\left(\alpha_{1}, \alpha_{2}, a, b\right)\right]$ such that

$$
\operatorname{tg}_{t}\left(\alpha_{1} g_{t}^{-1}, \alpha_{2} g_{t}^{-1}\right)=\left(\alpha_{1}, \alpha_{2}\right),\left(t A_{t} a g_{t}^{-1}, t g_{t} b A_{t}^{-1}\right)=(a, b),
$$

for all $t=e^{i \theta}$. (The circle action naturally lifts up to the $\mathbb{Z}_{2}$ factor.) By appropriate conjugation (or equivalently by choosing appropriate representative $\left.\left(\alpha_{1}, \alpha_{2}, a, b\right)\right)$ we can assume

$$
g_{t}=\operatorname{diag}\left(t^{y_{1}}, \ldots, t^{y_{k}}\right) .
$$

Where each $y_{i}$ is an integer multiple of $\frac{1}{2}$. Assume there are $r$ distinct weights $z_{i}$ among these $y_{i}$ 's. We shall denote the multiplicity of $z_{i}$ by $m_{i}$. Without loss of generality we can also assume $A_{t}=\operatorname{diag}\left(t^{s}, t^{-s}\right)$ for $s$ being a nonnegative integer multiple of $\frac{1}{2}$ since these are the only one parameter subgroups of $S U(2) / \mathbb{Z}_{2}$ up to conjugation. Write all the matrices in block forms accordingly, i.e. let $\alpha_{l}(i j)$ denote the $(i, j)$ block of $\alpha_{l}$ and let $a(i)$ and $b(i)$ be the $i$ th column-wise block of $a$ and the $i$ th row-wise block of $b$ respectively, we have from (1.3) that

$$
\begin{aligned}
\left(1+z_{i}-z_{j}\right) \alpha_{l}(i j) & =0, \\
\left(\begin{array}{cc}
s+1-z_{i} & 0 \\
0 & -s+1-z_{i}
\end{array}\right) a(i) & =0 \\
b(i)\left(\begin{array}{cc}
s-1-z_{i} & 0 \\
0 & -s-1-z_{i}
\end{array}\right) & =0
\end{aligned}
$$

which clearly yields

$$
\begin{aligned}
\alpha_{l}(i j)=0 & \text { if } \quad\left(1+z_{i}-z_{j}\right) \neq 0 . \\
a(i)=0 & \text { if } \quad\left(s+1-z_{i}\right)\left(-s+1-z_{i}\right) \neq 0, \\
b(i)=0 & \text { if } \quad\left(-s+1+z_{i}\right)\left(s+1+z_{i}\right) \neq 0 .
\end{aligned}
$$

Define $z_{\min }$ to be the minimum of $z_{i}$ 's and that $z_{\max }$ be to the maximum of $z_{i}$ 's. For convenience, put the $z_{i}$ in descending order. we then have

Case 1. $z_{\min }<-s+1$ or $z_{\max }>s-1$. If $z_{i}=z_{\min }$, then $a(i)=0$ and $\alpha_{l}(i j)=0$ for all $j$ and $l=1,2$. Thus the rank condition (1.2) fails. The same argument applies to the other case. So there is no fixed point in this case. 
Case 2. $z_{\min }=1$ and $z_{\max }=1$. From (1.5) we see that $\alpha_{1}=\alpha_{2}=0$, and hence $b a=0$. If $k>1$, the rank condition can't be satisfied. So there is no fixed point in this case either. This leaves us with

Case 3. $z_{\min } \geq-s+1, z_{\max } \leq s-1$ and $s>1$ (or $s \geq 3 / 2$ ). Once again from 1.5 we know that the first row and the last column of both $\alpha_{1}$ and $\alpha_{2}$ are zeros. So in order to fulfil the rank condition the last column $a_{k}$ of $a$ and the first row $b_{1}$ of $b$ can't be zeros. This implies that $z_{\text {min }}=-s+1$ and $z_{1}=s-1$. Moreover, we see from (1.4) that there is exactly one linearly independent solution for each $a_{k}$ and $b_{1}$ respectively, and that $i$ th column $a_{i}$ of $a$ is zero if $i \neq k$ and that $i$ th row $b_{i}$ of $b$ is zero if $i \neq 1$. The rank condition then implies that the multiplicities $m_{1}=m_{r}=1$. Now from 1.5 and the rank condition again we obtain (by bootstrapping) $z_{i+1}=z_{i}-1$, i.e. $z_{\max }=z_{1}=s-1, z_{2}=s-2, \ldots, z_{2 s-1}=z_{\min }=-s+1$. Thus the only possible nonzero blocks of $\alpha_{l}(i j)$ are $\alpha_{l}(i+1, i)$, the ones just below the diagonal blocks. They satisfy the following equations

$$
\alpha_{1}(i+1, i) \alpha_{2}(i, i-1)=\alpha_{2}(i+1, i) \alpha_{1}(i, i-1)
$$

for $i=2, \ldots, 2 s-2$. Note also that the number of distinct $z_{i}$ are $r=2 s-1$ which can realize any integer greater than one. Hence $s \geq 1$. Now let's study the last case more closely. From now on, we take $s=p / 2$ for $p$ being an natural numbers greater than two.

As we have just seen that the fixed point set is the union of a finite number of subsets indexed by the sequences $\left(m_{1}=1, m_{2}, \ldots, m_{2 s-2}, m_{2 s-1}=\right.$ $1)$, here $m_{i}$ are natural numbers. We shall denote them by $F\left(m_{1}, \ldots, m_{2 s-1}\right)$. The first observation is that any two of them if not empty are disconnected. One can see this as follows. First of all these sets are closed. To see that they are disjoint, consider the sets $F\left(m_{1}, \ldots, m_{2 s-1}\right)$ and $F\left(m_{1}^{\prime}, \ldots, m_{2 s^{\prime}-1}^{\prime}\right)$. Let $j>1$ be the smallest integer such that $m_{i} \neq m_{i}^{\prime}$, and let's assume that $m_{j}<m_{j}^{\prime}$. If there is a point in the intersection of the two sets, then the row $\left(\sum_{i=1}^{j} m_{i}\right)+1$ of both $\alpha_{l}$ are identically zeros by just comparing the block shapes of the two matrices. This contradicts (1.2). To summarize, we have

Lemma 1.7. The fixed point set of $\mathcal{M}_{k}(2)$ can be written as a finite number of disconnected union of smooth manifolds $F\left(m_{1}, \ldots, m_{2 s-1}\right)$ with $\sum_{i=1}^{2 s-1} m_{i}=k . F$ is either empty or is the quotient of the set of matrices $\left\{\left(\alpha_{1}(i+1, i), \alpha_{2}(i+1, i), z, w\right) \in M_{m_{i+1} \times m_{i}}(\mathbb{C}) \times M_{m_{i+1} \times m_{i}}(\mathbb{C}) \times \mathbb{C}^{*} \times \mathbb{C}^{*} ;\right.$ $i=1, \ldots, 2 s-2\}$ satisfying:

$$
\alpha_{1}(i+1, i) \alpha_{2}(i, i-1)=\alpha_{2}(i+1, i) \alpha_{1}(i, i-1)
$$




$$
\begin{aligned}
& \operatorname{rank}\left(\alpha_{1}(i+1, i) \quad \alpha_{2}(i+1, i)\right)=m_{i+1}, \\
& \operatorname{rank}\left(\begin{array}{c}
\alpha_{1}(i+1, i) \\
\alpha_{2}(i+1, i)
\end{array}\right)=m_{i}
\end{aligned}
$$

by the action of $\left(G L\left(m_{1}, \mathbb{C}\right) \times \cdots \times G L\left(m_{2 s-1}, \mathbb{C}\right)\right) \times_{\mathbb{Z}_{2}} T$ :

$$
\begin{gathered}
\alpha_{l}(i+1, i) \mapsto g(i+1) \alpha_{l}(i+1, i) g(i)^{-1}, \quad\left(\begin{array}{l}
0 \\
z
\end{array}\right) \mapsto t\left(\begin{array}{l}
0 \\
z
\end{array}\right) g(2 s-1)^{-1}, \\
\left(\begin{array}{ll}
w & 0
\end{array}\right) \mapsto g(1)\left(\begin{array}{ll}
w & 0
\end{array}\right) t
\end{gathered}
$$

for $(g(1), \ldots, g(2 s-1)) \in G L\left(m_{1}, \mathbb{C}\right) \times \cdots \times G L\left(m_{2 s-1}, \mathbb{C}\right)$, and $t \in T$.

Proof. (1.8) and (1.9) trivially follow from (1.1) and (1.2) respectively. (1.5) implies that the only nonzero blocks in $a$ and $b$ are $a(2 s-1)=\left(\begin{array}{l}0 \\ z\end{array}\right)$ and $b(1)=\left(\begin{array}{ll}w & 0\end{array}\right)$ respectively. It is simple to check that the subgroup of $S U(2)$ that acts on the set is the diagonal circle action described in the lemma. If $F\left(m_{1}, \ldots, m_{2 s-1}\right)$ is nonempty, then (1.9) implies that the subgroup of $G L(k, \mathbb{C})$ that preserves the shapes of $a, b$ and $\alpha_{l}$ is precisely the diagonal block matrix. Since the circle action is smooth (on a smooth manifold), each connected component of fixed point set $F$ is smooth. An easy alternative way to see this is to solve the equations (1.8) inductively (i.e. using inductive fibrations). If we start with the first set of equations, i.e. when $i=2$, thinking of $\alpha_{l}(3,2)$ as unknowns, then there is no restriction on $\alpha_{l}(2,1)$ other than the rank constraints. Thus the set of the pair $\alpha_{l}(2,1)$ forms a smooth affine variety. Moreover, since the $\operatorname{rank}\left(\begin{array}{c}\alpha_{2}(2,1) \\ \alpha_{1}(2,1)\end{array}\right)=m_{1}=$ 1 , the solution set $\left\{\left(\begin{array}{c}\alpha_{1}(3,2) \\ -\alpha_{2}(3,2)\end{array}\right)\right\}$ is a space $V_{2}$ of $m_{3}$ linearly independent frames in $\operatorname{Ker}\left(\begin{array}{l}\alpha_{2}(2,1) \\ \alpha_{1}(2,1)\end{array}\right)$, the kernel of the map $\left(\begin{array}{l}\alpha_{2}(2,1) \\ \alpha_{1}(2,1)\end{array}\right)$. Here the matrix acts on the vectors from the right. The correct solutions for $\alpha_{l}(3,2)$ are obtained from $V_{2}$ by removing some low dimensional varieties that violate the the other half of the rank condition. Again it is clear that the resulting set is a smooth complex affine variety. We do this inductively to the end in finite steps.

Now once again we take a circle action on $F$ and study its fixed point set. This time we let $T$ act "only" on $\alpha_{l}$ of $F$ as follows: $\alpha_{1} \mapsto t \alpha_{1}, \alpha_{2} \mapsto t^{-1} \alpha_{2}$.

Fact: If there is a $m_{i}$, other than $m_{1}$ and $m_{2 s-1}$, that equals 1 , then all $m_{i}=1$. Consequently, $F\left(m_{1}, \ldots, m_{2 s-1}\right)=F(1, \ldots, 1)=\mathbb{C P}^{1} \times \mathbb{R}^{+}$. 
The argument for this is very simple. Suppose that $m_{i}=1, m_{i+1}>1$ (The argument for $m_{i-1}>1$ is paralell.) for some $i \neq 1,2 s-1$. Then (1.6) implies that the column vectors $\alpha_{1}(i+1, i)$ and $\alpha_{2}(i+1, i)$ are linearly dependent and thus (1.2) is not satisfied. Thus all $m_{i}=1$. Moreover, either all $\alpha_{1}(i+1, i) \neq 0$ or all $\alpha_{2}(i+1, i) \neq 0$. Using the diagonal group action we can reduce it to the form that $\alpha_{l}=d_{l} D$, with the remaining action $H=\operatorname{diag}\left(\nu, \nu \mu, \nu \mu^{2} \ldots, \nu \mu^{k-1}\right)$. Where $d_{1}, d_{2}, \nu, \mu \in \mathbb{C}^{*},\left(d_{1}, d_{2}\right) \neq(0,0)$, and that $D$ is the matrix with 1 's below the diagonal and 0 's elsewhere. We can then use $H$ to put $z$ and $w$ in the lemma to be 1 , and there remains only $\mathbb{Z}_{2} \times T$ action with $\mathbb{Z}_{2}$ factor acting trivially (as is the case that center of $S U(2)$ acts trivially on $\mathcal{M}_{k}$ ). It is straight forward to check that it acts as $\left(d_{1}, d_{2}\right) \mapsto\left(d_{1} \mu, d_{2} \mu\right)$ for $\mu \in T$. This finishes the argument.

As a result, the fixed point set of $F(1, \ldots, 1)$ under the new circle action is the disjoint union of two copies of $\mathbb{R}^{+}$. Actually, above example can be easily generalized. We can give the following simple pictorial description. Let $G\left(m_{1}, m_{2}, \ldots, m_{2 s-1}\right)$ denote the graph in the plane by first connecting the points $(0,0),\left(1, m_{1}\right),\left(2, m_{2}\right), \ldots,\left(2 s-1, m_{2 s-1}\right),(2 s, 0)$ successively with the line segments and then closing it by the line segment between $(0,0)$ and $(2 s, 0)$. Then we have

Proposition 1.10. $F\left(m_{1}, m_{2}, \ldots, m_{2 s-1}\right)$ is nonempty if and only if $G\left(m_{1}, m_{2}, \ldots, m_{2 s-1}\right)$ is a trapezoid. In this case, the area of the trapezoid is precisely $k$. Moreover, the part of the fixed point set it represents is diffeomorphic to the disjoint union of two copies of $\mathbb{R}^{+}$if the graph is a honest trapezoid; to $\mathbb{R}^{+}$if the graph degenerates to a triangle.

Proof. If $m_{2}=1$, then all $m_{i}=1$ as we have just seen above. So assume $m_{2}>1$. But then $m_{2}=2$ from the rank condition (1.2). We can use the group action (the left part) to put $\alpha_{1}(2,1)=\left(\begin{array}{l}1 \\ 0\end{array}\right), \alpha_{2}(2,1)=\left(\begin{array}{l}0 \\ 1\end{array}\right)$. Choose a representative of any fixed point of the $T$ action of this form, there is an one parameter subgroup $G_{t}=\operatorname{diag}\left(g_{t}(1), g_{t}(2), \ldots, g_{t}(2 s-1)\right)$ of $G L\left(m_{1}, \mathbb{C}\right) \times \cdots \times G L\left(m_{2 s-1}, \mathbb{C}\right)$ such that

$$
\begin{gathered}
\operatorname{tg}(i+1) \alpha_{1}(i+1, i) g(i)^{-1}=\alpha_{1}(i+1, i), \\
t^{-1} g(i+1) \alpha_{2}(i+1, i) g(i)^{-1}=\alpha_{2}(i+1, i),
\end{gathered}
$$

for all $t \in T$. Using $\alpha_{l}(2,1)$ we have just derived, (1.11) implies that $g_{t}(2)=\left(\begin{array}{cc}t^{-1} & 0 \\ 0 & t\end{array}\right) g_{t}(1)$ 
Claim A: If $m_{i+1}>m_{i}$ for $i \leq l$, then $m_{i+1}=m_{i}+1=i+1$ for $i \leq l$. Moreover, the representatives of the fixed points can be chosen so that

$$
\alpha_{1}(i+1, i)=\left(\begin{array}{c}
I_{i} \\
0
\end{array}\right), \alpha_{2}(i+1, i)=\left(\begin{array}{c}
0 \\
I_{i}
\end{array}\right)
$$

and that

$$
g_{t}(i+1)=\operatorname{diag}\left(t^{-i}, t^{-i+2}, \ldots, t^{i}\right) g_{t}(1) .
$$

The claim is proved for $i=2$. Assume it is true for $i<l$. Then we shall show the claim for $i=l$ using induction. Write $\alpha_{1}(l+1, l)=$ $\left(\begin{array}{llll}c_{1} & c_{2} & \cdots & c_{l}\end{array}\right)$ and $\alpha_{2}(l+1, l)=\left(\begin{array}{llll}d_{1} & d_{2} & \cdots & d_{l}\end{array}\right)$, then (1.8) and the induction hypothesis imply that $\alpha_{2}(l+1, l)=\left(\begin{array}{lllll}c_{2} & c_{3} & \cdots & c_{l-1} & d_{l}\end{array}\right)$. Thus the rank of $\left(\alpha_{1}(l+1, l) \quad \alpha_{2}(l+1, l)\right)$ is at most $l+1$. By the assumption that $m_{l+1}>m_{l}$, it follows that $m_{l+1}=m_{l}+1$. So $c_{1}, c_{2}, \ldots, c_{l}, d_{l}$ are $l+1$ independent vectors. Moreover, (1.11) implies that $g_{t}(l+1)\left(\begin{array}{ccccc}c_{1} & c_{2} & \cdots & c_{l} & d_{l}\end{array}\right)=\left(\begin{array}{lllll}t^{-l} c_{1} & t^{-l+2} c_{2} & \cdots & t^{l-2} c_{l} & t^{l} d_{l}\end{array}\right) g_{t}(1)$. This verifies the claim $A$.

Since $m_{2 s-1}=1$, the sequence $m_{i}$ must stop increasing at some stage. Suppose it first happens at stage $j$, i.e. $m_{j-1}<m_{j} \geq m_{j+1}$, then we have

Claim B: If $j=m_{j}>m_{j+1}$, then $m_{j+1}=j-1$. Moreover, $m_{i+1}=m_{i}-1$ for $i>j$.

Following the notation of claim A, we see that exactly two of the vectors $c_{1}, c_{2}, \ldots, c_{j}, d_{j}$ have to be zeros since $g_{t}(j+1)$ can now have at most $j-1$ distinct eigenvalues and since the rank of $\left(\begin{array}{lllll}c_{1} & c_{2} & \cdots & c_{j} & d_{j}\end{array}\right)$ must be $j-1$. So we can choose $\left(\begin{array}{lllll}c_{1} & c_{2} & \cdots & c_{j} & d_{j}\end{array}\right)$ to be the identity matrix after deleting the two zero columns. We claim that $c_{1}=d_{j}=0$. Suppose the claim is false. We first assume that $c_{1} \neq 0$. So let the first zero column in $\left(\begin{array}{lllll}c_{1} & c_{2} & \cdots & c_{j} & d_{j}\end{array}\right)$ to be $c_{p}=0$ and the other one to be $c_{q}=0$. Here we use the notation that $c_{j+1}=d_{j}$. If $q=p+1$, then the matrix

$$
\left(\begin{array}{cccc}
c_{1} & c_{2} & \cdots & c_{j} \\
c_{2} & c_{3} & \cdots & c_{j+1}
\end{array}\right)
$$

has rank less than $j$ since its $p$ th column is zero. This clearly violates the condition (1.9). So we must have $q>p+1$. Repeat the above argument for the next level, i.e. for $\alpha_{1}(j+2, j+1)$ and $\alpha_{2}(j+2, j+1)$, we found that $\alpha_{1}(j+2, j+1)=\left(\begin{array}{lllll}c_{1} & c_{2} & \cdots & c_{j-1} & d_{j-1}\end{array}\right), \alpha_{2}(j+2, j+1)=$ $\left(\begin{array}{lllll}c_{2} & c_{3} & \cdots & c_{j-1} & c_{j}\end{array}\right)$. (Here the $c_{i}$ 's are different from the previous ones.) and that $c_{p}=c_{q-1}=0$. Thus inductively, we get to a situation where $q=p+1$ and derive the desired contradiction after $q-p$ steps. The argument for the other case when $c_{j+1} \neq 0$ is parelell. This proves the claim. 
Thus we can choose $\alpha_{1}(j+1, j)=\left(\begin{array}{ll}0 & I_{j-1}\end{array}\right)$ and $\alpha_{2}(j+1, j)=\left(\begin{array}{ll}I_{j-1} & 0\end{array}\right)$. The trivial induction shows that we can choose $\alpha_{1}(j+h, j+h-1)=$ $\left(\begin{array}{ll}0 & I_{j-h}\end{array}\right)$ and $\alpha_{2}(j+h, j+h-1)=\left(\begin{array}{ll}I_{j-h} & 0\end{array}\right)$ for $1 \leq h \leq j$. Moreover, we also find that $g_{t}(j+h)=\operatorname{diag}\left(t^{-j+h+1}, t^{-j+h+3}, \ldots, t^{j-h-1}\right) g_{t}(1)$ for $1 \leq h \leq j$.

Now we come to deal with the other case. First, we introduce a notation. Let $J^{j}$ denote the $j \times j$ matrix whose only nonzero entries are 1 's at $(i, i+1)$, and let $J_{j}$ denote the $j \times j$ matrix whose only nonzero entries are 1 's at $(i+1, i)$. Then we have

Claim C: If $j=m_{j}=m_{j+1}$, then for any $p \leq 1$ such that $m_{j+p}=$ $m_{j+1}, \alpha_{1}(j+p, j+p-1)$ and $\alpha_{2}(j+p, j+p-1)$ and $g_{t}(j+p)$ can be chosen to be either

$$
\alpha_{1}(j+p, j+p-1)=J^{j}, \alpha_{2}(j+p, j+p-1)=I_{j}, g_{t}(j+p)=t^{p} g_{t}(j)
$$

or

$$
\alpha_{1}(j+p, j+p-1)=I_{j}, \alpha_{2}(j+p, j+p-1)=J_{j}, g_{t}(j+p)=t^{-p} g_{t}(j) .
$$

Here we recall that $g_{t}(j)=\operatorname{diag}\left(t^{-j+1}, t^{-j+2}, \ldots, t^{j-1}\right) g_{t}(1)$.

From Claim A and its proof we have

$$
\alpha_{1}(j+1, j)=\left(\begin{array}{llll}
c_{1} & c_{2} & \cdots & c_{j}
\end{array}\right), \alpha_{2}(j+1, j)=\left(\begin{array}{llll}
c_{2} & c_{3} & \cdots & c_{j+1}
\end{array}\right),
$$

and

$g_{t}(j+1)\left(\begin{array}{lllll}c_{1} & c_{2} & \cdots & c_{j} & c_{j+1}\end{array}\right)=\left(\begin{array}{llll}t^{-j} c_{1} & t^{-j+2} c_{2} & \cdots & t^{j} c_{j+1}\end{array}\right) g_{t}(1)$.

So exactly one of the $c_{i}$ is zero for the same reason that rank $\left(\begin{array}{lllll}c_{1} & c_{2} & \cdots & c_{j} & c_{j+1}\end{array}\right)=j$ and that $g_{t}(j+1)$ has $j$ eigenvalues. Just as in the proof of Claim B, we can choose the matrix $\left(\begin{array}{lllll}c_{1} & c_{2} & \cdots & c_{j} & c_{j+1}\end{array}\right)$ to be the identity after deleting the zero column. It is a simple induction by appealing to (1.8) to show that as long as $m_{i}=m_{j}$ for some $i>j, \alpha_{1}(i, i-1)$ and $\alpha_{2}(i, i-1)$ can be chosen to be $\alpha_{1}(j, j-1)$ and $\alpha_{2}(j, j-1)$ respectively. Once $m_{i}$ begin to decrease, one more of the corresponding column vectors in $\left(\begin{array}{ccccc}c_{1} & c_{2} & \cdots & c_{q} & c_{q+1}\end{array}\right)$ has to be zero. Following the argument in $\mathrm{B}$, we find that it is either $c_{1}$ or $c_{q+1}$ that equals zero, i.e. no interior column vector can be zero. This proves the two choices for $\alpha_{1}(j+p, j+p-1)$ and $\alpha_{2}(j+p, j+p-1)$ in the claim. If $\alpha_{1}(j+p, j+p-1)=I_{j}$, then $g_{t}(j+p)=g_{t}(j+p) \alpha_{1}(j+p, j+p-1)=t^{-1} \alpha_{1}(j+p, j+p-1) g_{t}(j+p-1)=$ $t^{-1} g_{t}(j+p-1)$. If $\alpha_{2}(j+p, j+p-1)=I_{j}$, then $g_{t}(j+p)=t g_{t}(j+p-1)$. Inductively, this gives the result for $g_{t}(j+p)$ in both cases in the claim. 
Suppose $j+r$ is the last step such that $m_{j+r}=m_{j}$. Then we have Claim D: If

$$
\alpha_{1}(j+r, j+r-1)=J^{j}, \alpha_{2}(j+r, j+r-1)=I_{j}
$$

and

$$
g_{t}(j+r)=t^{r} \operatorname{diag}\left(t^{-j+1}, t^{-j+2}, \ldots, t^{j-1}\right) g_{t}(1),
$$

then we can choose

$\alpha_{1}(j+r+i, j+r+i-1)=\left(\begin{array}{ll}0 & I_{j-i}\end{array}\right), \alpha_{2}(j+r+i, j+r+i-1)=\left(I_{j-i}\right.$

and

$$
g_{t}(j+r+i)=t^{r} \operatorname{diag}\left(t^{-j+1+i}, t^{-j+3+i}, \ldots, t^{j-1-i}\right) g_{t}(1)
$$

for $1 \leq i \leq j-1$. If

$$
\alpha_{1}(j+r, j+r-1)=I_{j}, \alpha_{2}(j+r, j+r-1)=J_{j}
$$

and

$$
g_{t}(j+r)=t^{r} \operatorname{diag}\left(t^{-j+1}, t^{-j+2}, \ldots, t^{j-1}\right) g_{t}(1),
$$

then we can also choose

$\alpha_{1}(j+r+i, j+r+i-1)=\left(\begin{array}{ll}0 & I_{j-i}\end{array}\right), \alpha_{2}(j+r+i, j+r+i-1)=\left(I_{j-i}\right.$

but now $g_{t}(j+r+i)=t^{-r} \operatorname{diag}\left(t^{-j+1+i}, t^{-j+3+i}, \ldots, t^{j-1-i}\right)$.

Once again if we write $\alpha_{1}(j+r+1, j+r)=\left(\begin{array}{llll}c_{1} & c_{2} & \cdots & c_{j}\end{array}\right)$ and $\alpha_{2}(j+r+1, j+r)=\left(\begin{array}{lll}c_{2} & \cdots & c_{j+1}\end{array}\right)$ using (1.8), we find that in either case above both $c_{1}$ and $c_{j+1}$ have to be zero (Here we use again the crucial fact that no interior column vector $c_{i}$ can be zero as we proved). The rest of the proof is straightforward.

It is clear that the different representatives of $\alpha_{1}$ and $\alpha_{2}$ just described are in one to one correspondence to the different connected components of the fixed point set (Firstly, they don't intersect. Secondly, they are closed in the appropriate moduli spaces). Denote these connected components by $F F\left(\alpha_{1}, \alpha_{2}\right)$. The description of $g_{t}(i)$ implies that the isotropy group of $G L\left(m_{1}, \mathbb{C}\right) \times \cdots G L\left(m_{2 s-1}, \mathbb{C}\right)$ (as an action on $\left(\alpha_{1}, \alpha_{2}\right)$ part) at the given representatives $\left(\alpha_{1}, \alpha_{2}\right)$ above is always isomorphic to $\mathbb{C}^{*}$ embedded diagonally in $G L\left(m_{1}, \mathbb{C}\right) \times \cdots G L\left(m_{2 s-1}, \mathbb{C}\right)$ as $g_{1}(1) I_{k}$. Thus, $F F\left(\alpha_{1}, \alpha_{2}\right)$ is diffeomorphic to $\mathbb{R}^{+}$since the remaining action in (1.9) is

$$
(z, w) \mapsto\left(t g(1)^{-1} z, \operatorname{tg}(1) w\right) .
$$

This finishes the proof of the proposition.

From the proposition we see that $k=\sum_{i=1}^{2 s-1} m_{i}=j(j-1)+j(r+1)=$ $j(r+j)$. Hence, the number of the components $\mathbb{R}^{+}$of the fixed point set in $\mathcal{M}_{k}(2)$ is the same as the number of the divisors (factors) of the number $k$. Apply the Lefschetz fixed point theorem twice (in our application: the Euler characteristic of $\mathcal{M}_{k}(2)$ is the same as the Euler characteristic of the fixed point set of the circle action.) we have recovered a theorem of Furuta 
Theorem 1.12 (Furuta). $\chi\left(\mathcal{M}_{k}(2)\right)=E(k)$.

\section{The SU(3) case}

We shall now apply the same scheme to compute the Euler characteristics of $\mathcal{M}_{k}(3)$. First of all, we shall give a concrete description of the singularities of these moduli spaces. Geometrically, they are very easy to understand in this case. A singularity arises only if the corresponding gauge equivalent class of connections are reducible. The theorem of Donaldson tells that for each instanton there is a corresponding holomorphic bundle over $\mathbb{C P}^{2}$ which is trivial when it is restricted to a generic line in $\mathbb{C P}^{2}$. A connection is reducible if and only if its corresponding holomorphic bundle splits (holomorphically). Thus, unless the bundle is trivial to begin with it can not be split into direct sum of line bundles. This also explains why the moduli spaces $\mathcal{M}_{k}(2)$ are smooth. So the only singularities arise in $\mathcal{M}_{k}(3)$ are where the corresponding bundles over $\mathbb{C P}^{2}$ split into the direct sum of $S L(2, \mathbb{C})$ bundle and a trivial line bundle, i.e. the singularities in $\mathcal{M}_{k}(3)$ are precisely $\mathcal{M}_{k}(2)$. Incidently, it shows that any $S U(n)$-instanton of charge one is reduced to a $S U(2)$-instanton. To put everything concretely in our setup, we have

Lemma 2.1. The singular set $S_{k}(3)$ of $\mathcal{M}_{k}(3)$ is precisely $\mathcal{M}_{k}(2)$, and an open neighborhood $N_{k}(3)$ of $S_{k}(3)$ in $\mathcal{M}_{k}(3)$ is hemoemorphic to a cone bundle with the fiber being the cone on a weighted projective space $\mathbb{C P}^{2 k-3}$ for $k>1$.

Proof. Suppose $\left[\left(\alpha_{1}, \alpha_{2}, a, b\right)\right]$ is a singular point of the moduli space $\mathcal{M}_{k}(3)$, then the isotropy group of the action $S U(3) \times_{\mathbb{Z}_{3}} G L(k, \mathbb{C})$ contains a finite abelian subgroup $\mathbb{Z}_{p}$, the $p$ th root of unity for some prime $p$. As in previous section, we can assume that the $\mathbb{Z}_{p}$ action is of diagonal form. So write the first factor of the action as

$$
A_{\omega}=\left(\begin{array}{ccc}
\omega^{r} & & 0 \\
& \omega^{-r+s} & \\
0 & & \omega^{-s}
\end{array}\right) .
$$

Where $\omega$ is a generator of the $p$ th root of the unity and that $r, s$ are positive integer multiples of $\frac{1}{3}$ respectively. It is very easy to check using block-form that the second factor $g_{\omega}$ of the action must be of the form

$$
g_{\omega}=\left(\begin{array}{ccc}
I_{1} \omega^{r} & & 0 \\
& I_{2} \omega^{-r+s} & \\
0 & & I_{3} \omega^{-s}
\end{array}\right)
$$


here $I_{1}, I_{2}$ and $I_{3}$ are identity matrices of appropriate sizes. Note that $\omega^{r}, \omega^{-r+s}$ and $\omega^{-s}$ can't be all distinct for then there would exist a nontrivial $U(1)$-instanton over $S^{4}$. Thus, exactly two of them are the same. For the same reason, $g_{\omega}$ must be a scalar multiple of the identity, i.e., two of the three $I_{i}$ are of size "zero". Thus, a row of $a$ and corresponding column of $b$ are zeros, which implies that $\left[\left(\alpha_{1}, \alpha_{2}, a, b\right)\right]$ is an element of $\mathcal{M}_{k}(2)$. Under the action of $S U(3)$, all these points are identified to those of $\mathcal{M}_{k}(2)$ naturally embedded in $\mathcal{M}_{k}(3)$. Moreover, the image of the natural inclusion of $\mathcal{M}_{k}(2)$ into $\mathcal{M}_{k}(3)$ is singular and isotropy group of each singular point is isomorphic to $U(1)$. This proves that the singular set of $\mathcal{M}_{k}(3)$ is the smooth manifold $\mathcal{M}_{k}(2)$. To study the structure of the neighborhood of the singular set, we go to the based moduli space $\mathcal{M}_{k}^{b}(3)$ where the inverse image of the (quotient) singular set is the union of the orbit spaces of $S U(3)$ acting on the image of $\mathcal{M}_{k}^{b}(2)$ in $\mathcal{M}_{k}^{b}(3)$. This set, denoted by $S_{k}^{b}(3)$, is smooth connected manifold with real dimension $8 k+4$ since $S_{k}^{b}(3)$ is the total space of the fibration over $S_{k}(3)$ with fiber a homogenous space $S U(3) / U(1) \times S U(2)$. Take a $S U(3) / \mathbb{Z}_{3}$ invariant metric on $\mathcal{M}_{k}^{b}(3)$ and take a $S U(3) / \mathbb{Z}_{3}$ invariant tubular neighborhood $N_{k}^{b}(3)$ of $\mathcal{M}_{k}^{b}(2)$ in $\mathcal{M}_{k}^{b}(3)$. Then the isotropy group $T_{x}=U(1)$ of $x \in \mathcal{M}_{k}^{b}(2)$ acts isometrically in the fiber normal to $\mathcal{M}_{k}^{b}(2)$ at $x$ with $x$ as the only fixed point of the action. Since the fiber is isomorphic to $\mathbb{C}^{2 k-2}$, its quotient by $T_{x}$ is thus a cone over a weighted projective space $\mathbb{C P}^{2 k-3}$. The compactness of $S U(3) / \mathbb{Z}_{3}$ and smoothness of its action ensures that the quotient space of $N_{k}(3)$, quotienting $N_{k}^{b}(3)$ by $S U(3) / \mathbb{Z}_{3}$, fibers over $\mathcal{M}_{k}(2)$ with fiber $\mathbb{C P}^{2 k-3}$.

Remark 2.2. One simple but useful fact out of the proof worth mentioning is that the quotient space of $\mathcal{M}_{k}(3)$ by $S U(3) / \mathbb{Z}_{3}$ has no orbifold singularities. In other words, the smallest possible isotropy group is $U(1)$. This turns out to be true in general. Thus the cone bundle structure stated in the lemma can also be generalized in certain sense although it becomes more complicated. Once again, this is just a restatement of the discussion made in the beginning of the section.

Now consider the fixed point set of the circle action to $\mathcal{M}_{k}^{*}(3)=\mathcal{M}_{k}(3) \backslash S_{k}(3)$ as in the introduction. Take $k>1$. The entire previous section can be adapted to current case with minor changes. So we shall be very brief. As in last section, the isotropy group of the lifted circle action of a fixed point takes the following simple form:

$$
\begin{aligned}
& g_{t} \times A_{t}= \\
& \left.\operatorname{diag}\left(t^{y_{1}}, \ldots, t^{y_{k}}\right) \times \operatorname{diag}\left(t^{r}, t^{-r+s}, t^{-s}\right) \in G L(k, \mathbb{C}) \times_{\mathbb{Z}_{3}} S U(3)\right) .
\end{aligned}
$$

Where $r, s \geq 0$ and $y_{i}$ are integer multiples of $\frac{1}{3}$. Again, assume that $y_{i}$ 's 
are in decreasing order, and denote the distinct $y_{i}$ 's by $z_{\max }=z_{1}>z_{2}>$ $z_{3}>\cdots>z_{\min }$ and associated multiplicities by $m_{1}, m_{2}, \ldots$ Thus we have, $\left(z_{\max }, z_{\min }\right)=(r-1,-s+1)$ if $r+s>1$, or $(r-1,-r+s+1)$ if $2 r \geq s+2$, or $(-r+s-1,-s+1)$ if $2 s \geq r+2$ by rank consideration. Moreover, $z_{i}=z_{i+1}+1$ in order to satisfy the rank condition. This is very easy to justify: suppose that $z_{i_{0}}$ is the first $z_{i}$ such that $z_{i}+1 \neq z_{i-1}$, then we must have $a\left(i_{0}\right)$ and $b\left(i_{0}\right)$ both have maximal possible ranks (which is impossible as one of them is zero if applying second circle action), or $z_{i_{0}+1}+1=z_{i_{0}-1}$. In the latter situation, we have $m_{\text {last }}>1$. So a (last) would have rank at least two which is again impossible.

Remark 2.4. As we can see by now that the relations among $r, s$ and $z_{i}$ are very rigid for $S U(2)$ and $S U(3)$ which enable us to control the size of $\alpha_{i}$ (i.e. $m_{i}$ ) by $r$ and $s$. However, these relations become loser as $n$ increases (for $S U(n)$ ). For example, the relations $z_{i}=z_{i+1}+1$ are no longer valid if $n>3$. Nevertheless, the combinatorics involved is not terribly complicated.

Case I. $2 s=r$. In this case, $\left(z_{\max }, z_{\min }\right)=(2 s-1,-s+1),\left(r \geq \frac{2}{3}\right)$. all entries of $b$ are zero except its first row which is of the form $b(1)=$ ( $\left.\begin{array}{lll}c & 0 & 0\end{array}\right)$ for $c \in \mathbb{C}^{*}$, and all entries of $a$ are zero except the last (block) two columns. (Since these data "define" $S U(3)$-irreducible connections.) Moreover, $a(3 s-1)$, the last block of $a$, has rank two and is of the form: $a(3 s-1)=\left(\begin{array}{cc}0 & 0 \\ * & * \\ * & *\end{array}\right)$. Similar results hold if $2 r=s$. Apply second circle action, we find that the following analogue of (1.10):

Lemma 2.5. Under the above assumption that $2 s=r$ or $(2 r=s)$, the fixed point set of the circle actions consists of $F^{1}\left(1=m_{1}, m_{2}, \ldots, m_{3 s-1}=\right.$ 2) $\left(\right.$ or $\left.F^{2}\left(2=m_{1}, m_{2}, \ldots, m_{3 r-1}=1\right)\right)$ so that $G\left(m_{1}, \ldots, m_{3 s-1}, 1\right)$ (or $G\left(1, m_{1}, \ldots, m_{3 s-1}\right)$ (defined in last section) is a trapezoid. Moreover each of the $F^{1}$ (or $F^{2}$ ) is diffeomorphic to $\mathbb{R}^{4}$.

The only part need to be checked is that each $F^{1}\left(F^{2}\right)$ is diffeomorphic to $\mathbb{R}^{4}$. As we have seen in last section, the isotropy group of a fixed representative $\alpha_{1}, \alpha_{2}$ of such a fixed point is $\mathbb{C}^{*}$ diagonally embedded in $G L(k, \mathbb{C})$. So each of the $F^{1}$ is diffeomorphic to the quotient $\mathbb{C}^{*} \times G L(2, \mathbb{C})$ by $\mathbb{C}^{*} \times U(2)$ since the set consisting of $a(3 s-1)$ is diffeomorphic to $G L(2, \mathbb{C})$ (for $2 s=r$ ). Direct check of the action reveals that the quotient is diffeomorphic to $\left(\mathbb{R}^{+}\right)^{2} \times \mathbb{C}$ which is diffeomorphic to $\mathbb{R}^{4}$.

Case II. $2 s \neq r$ and $2 r \neq s$. If $\left(z_{\max }, z_{\min }\right)=(r-1,-r+s+1)$, then $\operatorname{rank}(a)=\operatorname{rank}(b)=1$. Thus the fixed point represents a reducible $S U(2)$ connection. The same is true when $\left(z_{\max }, z_{\min }\right)=(-r+s-1,-s+1)$. There remains one more case to consider: $\left(z_{\max }, z_{\min }\right)=(r-1,-s+1)$. 
In this case, the only nonzero blocks of $a$ and $b$ are the last column of $a$ which is of the form $a($ last $)=\left(\begin{array}{l}0 \\ 0 \\ *\end{array}\right)$, the first row of $b$ which is of the form $b(1)=\left(\begin{array}{lll}* & 0 & 0\end{array}\right)$, and two more possible nonzero blocks: $a\left(i_{0}\right)=$ $\left(\begin{array}{l}0 \\ * \\ 0\end{array}\right)$ (corresponding to $\left.z_{i_{0}}=-r+s+1\right)$ and $b\left(i_{0}+2\right)=\left(\begin{array}{lll}0 & * & 0\end{array}\right)$ (corresponding to $z_{i_{0}+2}=-r+s-1$ ). We shall now show that both $a_{i_{0}}$ and $b\left(i_{0}+2\right)$ must be zero blocks. This then implies that the resulting fixed points if exist must lie in $S_{k}(3)$. To this end, we explore the second circle action in more details. Again we may assume the isotropy group of a fixed point is of the form 2.3. Then we have: $z_{1}=r-1, z_{l a s t}=z_{r+s-1}=-s+1$. Suppose $a\left(i_{0}\right) \neq 0$, or $b\left(i_{0}+2\right) \neq 0$, then we must also have $z_{i_{0}}=-r+s$, thus also $i_{0}=2 r-s$. (Implicitly, this implies that $2 r>s+1$ and $2 s>r+1$.) Two simple but useful facts out of the proof of last section worth stating here:

II a). Up to the factor $g_{t}(1)$ (which is $t^{r}$ in present situation), the weights of representation of each $g_{t}(i)$ obtained from the isotropy representation $g_{t}$ described above are of same parities, i.e. either all even or all odd.

II b). After factoring out $g_{t}(1), g_{t}(i)$ has weights bounded by $i-1$ from above and by $-i+1$ from below.

We shall assume $m_{1}=1$. Start the induction proceedure as in last section with $i=1,2, \ldots$, the results are identical up to $i=i_{0}$ (the equality here is important). Since the induced action on $a\left(i_{0}\right)$ is $t^{s-r} a\left(i_{0}\right) g^{-1}\left(i_{0}\right)$, $a\left(i_{0}\right)$ has to be zero for it being fixed by the action using II a) and the fact above that $i_{0}=2 r-s$. By inspecting the induced action on $b\left(i_{0}+2\right)$ and using II b), we find that $b\left(i_{0}+2\right)=0$. For $m_{1}=2$, note that $m_{\text {last }}=1$, so we can use backward induction to obtain the same result. To summarize, we have following

Theorem 2.6. $\chi\left(\mathcal{M}_{k}(3)\right)=2 E(k+1)+(2 k-3) E(k)-4$ for $k>1$.

The proof is obvious by combining the fixed point theorem and MayerVietoris principal and by using the results of lemma 2.1 and 2.5 which yields

$$
\begin{aligned}
& \chi\left(\mathcal{M}_{k}(3)\right)=\chi\left(\mathcal{M}_{k}^{*}(3)\right)+\chi\left(N_{k}(3)\right)-\chi\left(\mathcal{M}_{k}(2)\right)= \\
& 2(E(k+1)-2)+(2 k-2) E(k)-E(k)=2 E(k+1)+(2 k-3) E(k)-4 .
\end{aligned}
$$

\section{Compactifications}

Let $\chi_{c}(M)$ denote the Euler characteristic of $M$ with compact support. Since $\chi_{c}(\mathbb{R})=-1$ and $\chi_{c}\left(\mathbb{R}^{4}\right)=1$ and since the fuctor $\chi_{c}$ is additive on 
disjoint sets, (Author is thankful to M. Goresky for pointing out of this fact.) we have, for $k>0$, (Note that the following formulas are also valid for $k=1$.)

$$
\begin{aligned}
& \chi_{c}\left(\mathcal{M}_{k}(2)\right)=-E(k), \quad \chi_{c}\left(\mathcal{M}_{k}(3)\right)= \\
& \chi_{c}\left(\mathcal{M}_{k}^{*}(3)\right)+\chi_{c}\left(\mathcal{M}_{k}(2)\right)=2 E(k+1)-E(k)-4
\end{aligned}
$$

Denote by $\operatorname{Sym}^{n}\left(S^{4}\right)$ the $n$-fold symmetric product of four sphere. Let $\overline{\mathcal{M}}_{k}^{U}(2)=\coprod_{i=0}^{k} \mathcal{M}_{i}(2) \times \operatorname{Sym}^{k-i}\left(S^{4}\right)$ be the Uhlenbeck compactification of $\mathcal{M}_{k}(2)$. Here $\mathcal{M}_{0}(2)$ and $\operatorname{Sym}^{0}\left(S^{4}\right)$ are meant to be singleton sets. So we shall define $E(0)=1$. We have

$$
\begin{aligned}
& \chi\left(\overline{\mathcal{M}}_{k}^{U}(2)\right)=\chi_{c}\left(\overline{\mathcal{M}}_{k}^{U}(2)\right)=\sum_{i=0}^{k} \chi_{c}\left(\mathcal{M}_{i}(2)\right) \chi_{c}\left(\operatorname{Sym}^{k-i}\left(S^{4}\right)\right)= \\
& \chi\left(\operatorname{Sym}^{k}\left(S^{4}\right)\right)+\sum_{i=1}^{k}(-E(i)) \chi\left(\operatorname{Sym}^{k-i}\left(S^{4}\right)\right)= \\
& (k+1)-\sum_{i=1}^{k} E(i)(k-i+1) .
\end{aligned}
$$

As the singularity set of $\mathcal{M}_{k}(3)$ is $\mathcal{M}_{k}(2)$, we still have the following Uhlenbeck compactfications for $\mathcal{M}_{k}(3): \overline{\mathcal{M}}_{k}^{U}(3)=\coprod_{i=0}^{k} \mathcal{M}_{i}(3) \times \operatorname{Sym}^{k-i}\left(S^{4}\right)$. Thus we have

$$
\begin{aligned}
& \chi\left(\overline{\mathcal{M}}_{k}^{U}(3)\right)=\chi_{c}\left(\overline{\mathcal{M}}_{k}^{U}(3)\right)=\sum_{i=0}^{k} \chi_{c}\left(\mathcal{M}_{i}(3)\right) \chi_{c}\left(\operatorname{Sym}^{k-i}\left(S^{4}\right)\right)= \\
& \chi\left(\operatorname{Sym}^{k}\left(S^{4}\right)\right)+\sum_{i=1}^{k}[2 E(i+1)-E(i)-4] \chi_{c}\left(\operatorname{Sym}^{k-i}\left(S^{4}\right)\right)= \\
& k+1+\sum_{i=1}^{k}[2 E(i+1)-E(i)-4](k-i+1), \text { for } k>1 .
\end{aligned}
$$

Here we have used the fact that $\mathcal{M}_{1}(3)=\mathcal{M}_{1}(2)$.

Finally, we shall calculate the Euler characteristics of the moduli spaces under bubble tree compactifications [PW, Y]. Note the following simple fact: the Euler characteristics of the configuration space of three or more distinct points over four sphere is zero. Thus the bubble trees whose corresponding strata give nontrivial contributions to the Euler characteristics 
are of the form $\mathcal{T}_{k}(l, m)$. Here $\mathcal{T}_{k}(l, m)$ stands for the bubble tree with at most two stacks of four spheres over the base four sphere of length $l$ and $m$ respectively. Moreover, the corresponding stratum $M_{\mathcal{T}_{k}(l, m)}$ belongs to the bubble tree compactification $\overline{\mathcal{M}}_{k}^{B}$ of $\mathcal{M}_{k}$. So $l, m$ are nonnegative integers whose sum is less than or equal to $k$. We have

$$
\begin{aligned}
& \chi_{c}\left(M_{\left.\mathcal{T}_{k}(l, m)\right)}(2)\right)= \\
& \chi_{c}\left(\mathcal{M}_{k-l-m}(2) \times\left(S^{4} \times \mathbb{R}^{4(l-1)} \times \mathbb{R}^{4 m}\right)\right)=2 \chi_{c}\left(\mathcal{M}_{k-l-m}(2)\right)
\end{aligned}
$$

if $l>m$, and

$\chi_{c}\left(M_{\left.\mathcal{T}_{k}(l, l)\right)}(2)\right)=\chi_{c}\left(\mathcal{M}_{k-2 l}(2) \times\left(\left(S^{4} \times \mathbb{R}^{4(l-1)}\right) \times_{\mathbb{Z}_{2}} \mathbb{R}^{4 l}\right)\right)=\chi_{c}\left(\mathcal{M}_{k-2 l}(2)\right)$.

Thus we have

$$
\begin{aligned}
\chi\left(\overline{\mathcal{M}}_{k}^{B}(2)\right)= & \sum_{l=1}^{[k / 2]} \sum_{m=0}^{l-1} 2 \chi_{c}\left(\mathcal{M}_{k-l-m}(2)\right)+\sum_{l=[k / 2]+1}^{k} \sum_{m=0}^{k-l} 2 \chi_{c}\left(\mathcal{M}_{k-l-m}(2)\right) \\
& +\sum_{l=0}^{[k / 2]} \chi_{c}\left(\mathcal{M}_{k-2 l}(2)\right) .
\end{aligned}
$$

Entire analogously, we have

$$
\begin{aligned}
\chi\left(\overline{\mathcal{M}}_{k}^{B}(3)\right)= & \sum_{l=1}^{[k / 2]} \sum_{m=0}^{l-1} 2 \chi_{c}\left(\mathcal{M}_{k-l-m}(3)\right)+\sum_{l=[k / 2]+1}^{k} \sum_{m=0}^{k-l} 2 \chi_{c}\left(\mathcal{M}_{k-l-m}(3)\right) \\
& +\sum_{l=0}^{[k / 2]} \chi_{c}\left(\mathcal{M}_{k-2 l}(3)\right) .
\end{aligned}
$$

To summarize, we have 


\section{Proposition 3.1.}

a) $\chi\left(\overline{\mathcal{M}}_{k}^{U}(2)\right)=k+1-\sum_{i=1}^{k} E(i)(k-i+1)$, for $k>0$.

b) $\chi\left(\overline{\mathcal{M}}_{k}^{U}(3)\right)=k+1+\sum_{i=1}^{k}[2 E(i+1)-E(i)-4](k-i+1)$, for $k>1$.

c1) $\chi\left(\overline{\mathcal{M}}_{2 k}^{B}(2)\right)=2 k+1-\sum_{l=1}^{k} \sum_{m=0}^{l-1} 2 E(2 k-l-m)-$

$$
\sum_{l=1}^{k} \sum_{m=0}^{k-l-1} 2 E(k-l-m)-\sum_{l=0}^{k-1} E(2 k-2 l), \text { for } k>0 .
$$

c2) $\chi\left(\overline{\mathcal{M}}_{2 k}^{B}(3)\right)=-8 k^{2}-2 k+1+$

$$
\begin{aligned}
& 2 \sum_{l=1}^{k} \sum_{m=0}^{l-1}(2 E(2 k-l-m+1)-E(2 k-l-m))+ \\
& 2 \sum_{l=1}^{k} \sum_{m=0}^{k-l-1}(2 E(k-l-m+1)-E(k-l-m))+ \\
& \sum_{l=0}^{k-1}(2 E(2 k-2 l+1)-E(2 k-2 l)) \text { for } k>0 .
\end{aligned}
$$

d1) $\chi\left(\overline{\mathcal{M}}_{2 k+1}^{B}(2)\right)=2 k+2-\sum_{l=1}^{k} \sum_{m=0}^{l-1} 2 E(2 k+1-l-m)-$

$$
\sum_{l=1}^{k+1} \sum_{m=0}^{k-l} 2 E(k+1-l-m)-\sum_{l=0}^{k} E(2 k+1-2 l), \text { for } k>0 .
$$

d2) $\chi\left(\overline{\mathcal{M}}_{2 k+1}^{B}(3)\right)=-8 k^{2}-10 k-2+$

$$
\begin{aligned}
& 2 \sum_{l=1}^{k} \sum_{m=0}^{l-1}(2 E(2 k-l-m+2)-E(2 k+1-l-m))+ \\
& 2 \sum_{l=1}^{k} \sum_{m=0}^{k-l}(2 E(k-l-m+2)-E(k+1-l-m))+ \\
& \sum_{l=0}^{k}(2 E(2 k-2 l+2)-E(2 k+1-2 l)) \text { for } k>0 .
\end{aligned}
$$




\section{Acknowledgements}

The author is grateful to Clifford Taubes for his support and very helpful conversations regarding the problem. The author also wishes to thank Aiko Liu, Kefeng Liu, Tomasz Mrowka, Gang Tian and Weiping Zhang for helpful discussions and to Cumrun Vafa and Edward Witten for bringing the reference $[\mathrm{F}]$ to his attention. Finally, the author wishes to thank the Department of Mathematics at Harvard University for its hospitality where a large part of the note was done.

\section{References}

[BHMM] C. P. Boyer, J. C. Hurtubise, B. M. Mann, and R. J. Milgram, The topology of instanton moduli spaces. I: The Atiyah-Jones conjecture, Ann. of Math. 137 (1993), 561-609.

[CM] R. L. Cohen and R. J. Milgram, The homotopy type of gauge theoretic moduli spaces, MSRI Pub. 27 (1993), 15-55.

[D] S. K. Donaldson, Instantons and geometric invariant thoery, Comm. Math. Phys. 93 (1984), 453-461.

[F] M. Furuta, Euler number of moduli spaces of instantons, Proc. Japan Acad. Ser. A Math. Sci. 63 (1987), 266-267.

[K] F. Kirwan, Geometric invariant theory and Atiyah-Jones conjecture, Proc. S. Lie Mem. Conf., Scand. Univ. Press, 1994, pp. 161-188.

[PW] T. Parker and J. Wolfson, Pseudo-holomorphic maps and bubble trees, J. Geom. Anal. 3 (1993), 63-98.

[S] Marc Sanders, Classifying spaces and Dirac operators coupled to instantons, Trans. Amer. Math. Soc. 347 (1995), 4037-4072.

[T] C. H. Taubes, The stable topology of self-dual moduli spaces, J. Diff. Geom. 29 (1989), 163-230.

[Ti1] Youliang Tian, The based $S U(n)$-instanton moduli spaces, Math. Ann. 298 (1994), 117-139.

[Ti2] The Atiyah-Jones conjecture for classical groups and Bott periodicity, J. Diff. Geom. (to appear).

[VW] C. Vafa and E. Witten, A strong coupling test of S-duality, Nucl. Phys. B431 (1994), 3-77.

[Y] R. Ye, Gromov's compactness theorem for pseudo-holomorphic curves, Trans. Amer. Math. Soc. 342 (1994), 671-694.

Courant Institute of M athematical Sciences, 251 Mercer Street, New Y ORK, N Y 10012

E-mail address: ytian@cims.nyu.edu 